\title{
Bio-Chemo-Opto-Mechanical (BioCOM) Sensors for Real-Time Characterization for D\&D Applications (82799)
}

\section{Research Objective}

Basic and applied research is being conducted to develop simple to use chemical and biological sensor chips utilizing bio-chemo-mechanics for realtime, in-situ, detection of technetium, mercury, uranium, copper, and lead for deactivation and decommissioning applications. The bio-chemo-opto-mechanical (Bio-COM) chip involves properly fashioned arrays of micromachined silicon cantilevers containing embedded deformable diffraction gratings functionalized with chemically selective coatings. Adsorption of specific molecules on the cantilever array leads to bending, which changes the diffraction of light from the array. The biochemo-opto-mechanical (BioCOM) chips will be designed to contain an array of pixels, with each pixel containing an array of microcantilever springs in which one surface is derivatized with either an antibody coating or a self-assembled monolayer (SAM) coating for detecting $\mathrm{Hg}(\mathrm{II})$, $\mathrm{Hg}(0), \mathrm{Cu}(\mathrm{II}), \mathrm{Pb}(\mathrm{II}), \mathrm{U}(\mathrm{VI})$, or $\mathrm{TcO}_{4}{ }^{-}$. The BioCOM sensor platform also offers the advantage of simultaneous measurement of many analytes using a single chip. The readout mechanism can be a reflected laser beam, producing a diffraction pattern, or in an ideal case the diffraction of daylight resulting in a change of color. In the latter case the proposed sensors would not require any external power, external or on-board electronics, or fluorescent dyes and associated optics, which will keep its fabrication and operation costs low while making it simple to use for realtime environmental monitoring.

\section{Research Progress and Implications}

This report summarizes the work in the first 20 months (June, 2003) of a three year project. The basic goals of this program are the basic research to design and develop coatings to make selective sensors, the design and fabrication of the test chip and the optical detection system, and the design and development and field test of a prototype sensor. The latter general goal will be carried out in the third year of the project. Good progress has been made toward advancement of the first two goals.

Chip Design and Microfabrication and Optical Detection System Design. The design and fabrication of microcantilever sensors based on the concept of a deformable diffraction grating has been carried out in Professor Arun Majumdar's group at UC Berkeley. The original concept was an interdigitated array of cantilevers, fabricated by a surface micromachining process,

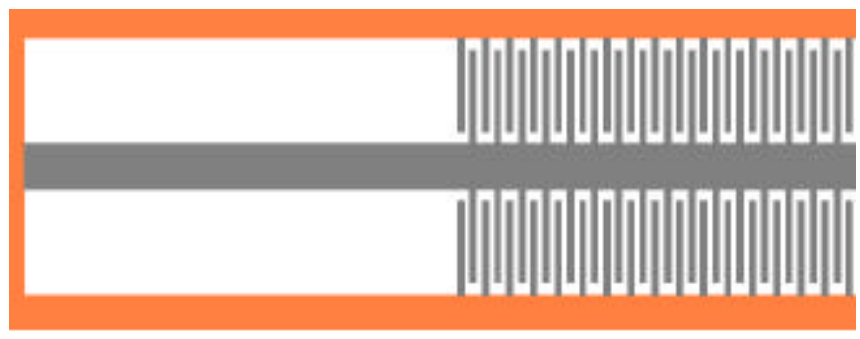
shown schematically in the figure to the left. The interdigitated fingers of the cantilevers on the chip collectively reflect incident light into diffraction patterns. However the cantilevers bend continuously and to a sufficient degree that multiple diffraction patterns, corresponding to different degrees of bending, made determination of the bending distance difficult. Work is in progress to develop a different style of deformable grating that will have surfaces that remain parallel for a single diffraction distance in an array. The new concept is shown in the following figures. 

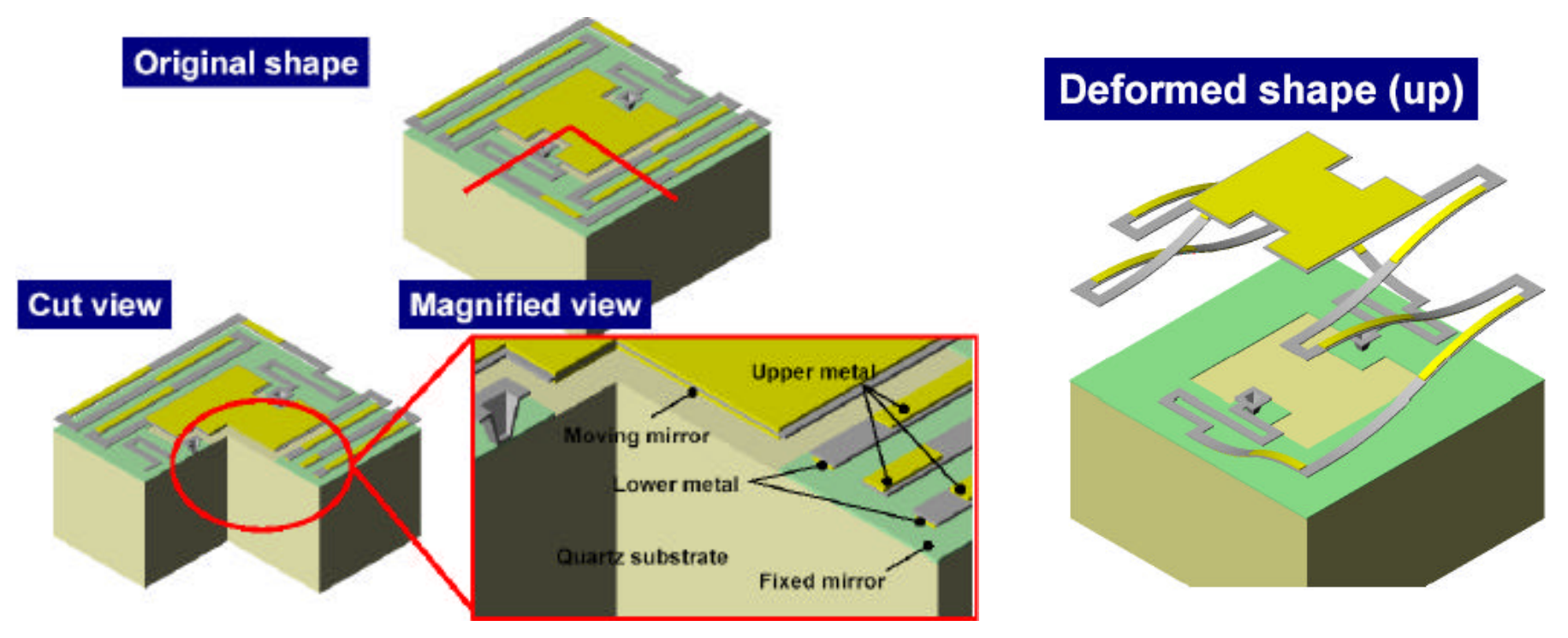

Parabolic microcantilever arms bend in opposition to one another so that the platform always remains parallel to the surface of the chip. In this case only a single diffraction pattern will be observed. Arrays of multiple sensing elements will be constructed on a single chip.

Design and Development of Selective Coatings. Work is in progress to adapt the surface chemistry to attach metal specific antibodies to the surface of the cantilever chips. Work is in progress to attach the ligand 2,9-dicarboxy-5-amino-1,10-phananthroline to the silicon surface of a microcantilever coated on one surface with gold. This ligand is moderately selective for U(VI), and Prof Diane Blake at Tulane University has developed an antibody for this metal ligand adduct. Tests are underway with traditional triangular AFM cantilevers having the surface modified with this chemistry, using both thiol derivatives for attachment to gold and silane reagents for attachment to the silicon surface. The modified cantilever is expected to bend when the antibody recognizes the metal-ligand complex attached to the surface. We have tested the attachment of quaternary ammonium salts, tethered to the surface of the silicon cantilevers with carbon silicon bonds. These groups will be selective for the pertechnetate and uranylcarbonate anions in solution. We have previously investigated triethylammoniumdodecane-11-thiol for attachment to the gold surface, but this compound spontaneously decomposes and will be unsuitable for long-term use. Treatment of silicon surface with $\mathrm{NH}_{4} \mathrm{~F}$ in aqueous solution generates $\mathrm{SiH}$ groups on the surface. When activated by UV light, $\mathrm{SiH}$ bonds undergo addition across carbon-carbon double bonds to produce robust and inert $\mathrm{Si}-\mathrm{C}$ bonds to the cantilever surface while preserving the quaternary ammonium site for molecular recognition. Initial work, conducted with triangular cantilevers, shows that the quaternary ammonium modified cantilever is selective for chromate ion as well as for the other species. The combination of micromechanical sensing with control of the electrochemical potential of the surface offers the promise for achieving chemical selectivity for sensor applications in liquid environments. Microcantilevers coated with gold have been used as the working electrode to measure the current-potential response and simultaneous bending characteristics by cyclic voltammetry in solutions of $\mathrm{NaNO}_{3}$ and $\mathrm{K} 33 \mathrm{Fe}(\mathrm{CN})_{6} / \mathrm{NaNO}_{3}$. The measured cantilever bending deflection is due to differential surface stress introduced by electrochemically-induced changes in the surface charge density, ion adsorption/desorption and the charge transfer on the electrode 
surface. The change in surface stress was can be used as used as the basis for measurement in an electrochemical sensor. Combining stress measurement with electrochemical technique, a microcantilever sensor was shown to have high sensitivity and selectivity for the detection of trace amounts $\left(10^{-11} \mathrm{M}\right)$ of $\mathrm{Cu}^{2+}$.

\section{Planned Activities}

In the remainder of this fiscal year, work will continue on observing deflection of a cantilever, modified by attachment of the phenanthroline derivative which is selective for U(VI), following interaction of antibodies with the surface. Investigations of the quaternary ammonium compound bound to the $\mathrm{Si}$ surface will be conducted with $\mathrm{ReO}_{4}{ }^{-}$, a non-radioactive analog of $\mathrm{TcO}_{4}{ }^{-}$. A method of electronically recording the diffraction patterns of the deformable grating as a function of time will be developed. Our goal is to conduct a field test of a $\mathrm{Hg}$ sensor based on the deformable grating at DOE's Y12 site next fiscal year.

Information Access - Publications in FY 03

"Observation of the Electrochemical Potential-Induced Surface Stress by Microcantilevers," F. Tian, J.H. Pei, D.L. Hedden, G.M. Brown and T. G. Thundat, submitted for publication.

"Allosteric Binding Properties of a Monoclonal Antibody and Its Fab Fragment," Robert C.

Blake II, James B. Delehanty, Mehraban Khosraviani, Haini Yu, R. Mark Jones, and

Diane A. Blake, Biochemistry 2003, 42, 497-508 